\title{
El ejercicio físico y el estado de ánimo en adolescentes
}

\section{Physical exercise and mood in adolescents}

1 Diego Javier Mayorga Ortiz

http://orcid.org/0000-0001-8201-3582

Universidad Técnica de Ambato, Facultad de Ciencias de la Educación, Carrera de Pedagogía de la Actividad Física y Deporte. Tungurahua, Ecuador,

dj.mayorga@uta.edu.ec

2 Luis Alfredo Jiménez Ruíz

https://orcid.org/0000-0001-8704-0563

Universidad Técnica de Ambato, Carrera de Pedagogía de la Actividad Física y Deporte, Tungurahua, Ecuador

la.jimenez@uta.edu.ec

3 Alicia Carolina Cevallos Torres

(iD) https://orcid.org/0000-0002-5373-6834

Pontificia Universidad Católica Sede Ambato, Posgrados Innovación en Educación.

Tungurahua, Ecuador

accevallost@pucesa.edu.ec

4 Javier Fernando Herrera Tapia

https://orcid.org/0000-0002-2654-0123

Universidad Técnica de Ambato, Facultad de Ciencias de la Educación, Carrera de

Pedagogía de la Actividad Física y Deporte. Tungurahua, Ecuador,

jherrera2209@uta.edu.ec

Artículo de Investigación Científica y Tecnológica

Enviado: 24/12/2021

Revisado: 29/12/2021

Aceptado: $12 / 01 / 2022$

Publicado:08/03/2023

DOI: https://doi.org/10.33262/concienciadigital.v6i1.4.2027

Cítese. Mayorga Ortiz, D. J., Jiménez Ruíz, L. A., Cevallos Torres, A. C., \& Herrera Tapia, J. F. (2023). El ejercicio físico y el estado de ánimo en adolescentes. ConcienciaDigital, 6(1.4), 766-780. https://doi.org/10.33262/concienciadigital.v6i1.4.2027

CONCIENCIA DIGITAL, es una Revista Multidisciplinar, Trimestral, que se publicará en soporte electrónico tiene como misión contribuir a la formación de profesionales competentes con visión humanística y crítica que sean capaces de exponer sus resultados investigativos y científicos en la misma medida que se promueva mediante su intervención cambios positivos en la sociedad. https://concienciadigital.org

La revista es editada por la Editorial Ciencia Digital (Editorial de prestigio registrada en la Cámara Ecuatoriana de Libro con No de Afiliación 663) www.celibro.org.ec 


\section{Palabras} claves: ejercicio físico, estado de ánimo, emociones, salud mental

\section{Keywords:}

physical exercise, mood, emotions, mental health
Resumen

La práctica de ejercicio físico presenta varios beneficios en la salud tanto física como mental. La investigación se centrará en esta última. Objetivo: En el presente trabajo investigativo se determina la relación entre el ejercicio físico y el estado de ánimo de los estudiantes de bachillerato de la Unidad Educativa 17 de abril de la provincia de Tungurahua, durante el periodo abril-agosto de 2021. Metodología: Se trata de una investigación cuantitativa, no experimental de alcance descriptivo con método del nivel teórico hipotético-deductivo. Para la recolección de datos se utilizaron dos instrumentos que permitieron medir cada variable en una muestra de estudio de 49 estudiantes perteneciente a segundo de bachillerato. Resultados: Los resultados de la investigación fueron comprobados a través de la prueba chi cuadrado con diferenciación asintótica bilateral en un nivel de $\mathrm{P} \leq 0.05$ dando como resultado un valor estadístico significativo en el nivel de asociación del ejercicio físico y el estado de ánimo de los estudiantes. Conclusión: Se concluye que el ejercicio físico si influye sobre el estado de ánimo. Cuando existe un nivel alto o moderado de práctica de ejercicio físico, el estado de ánimo del individuo será positivo, por el contrario, al haber un bajo o nulo nivel de práctica de ejercicio físico existirá una tendencia a que se desarrollen estados de ánimo negativos.

Abstract

The practice of physical exercise has several benefits in both physical and mental health. Research will focus on the latter. Objective: In this research work, the relationship between physical exercise and the state of mind of high school students of the Educational Unit April 17 of the province of Tungurahua is determined, during the period April-August 2021. Methodology: It is a quantitative, non-experimental, descriptive research with a hypothetical-deductive theoretical level method. For data collection, two instruments were used that made it possible to measure each variable in a study sample of 49 students belonging to the second year of high school. Results: The results of the research were verified through the chi-square test with bilateral asymptotic differentiation at a level of $\mathrm{P} \leq 0.05$, resulting in a significant statistical value in the level of association between physical exercise and the mood of the students. Conclusion: It is 
concluded that physical exercise does influence mood. When there is a high or moderate level of physical exercise, the individual's mood will be positive, on the contrary, when there is a low or no level of physical exercise there will be a tendency for negative moods to develop.

\section{Introducción}

El ejercicio físico es indispensable para gozar de un buen estado de salud. Bisquert et al. (2020) menciona que el ejercicio físico es una actividad que se planifica y se estructura con un objetivo en específico, constituyéndose como una herramienta preventiva de enfermedades no transmisibles, además de un protector de la salud física y mental pues contribuye a la condición física activa en las personas (Tobar et al., 2020). La salud mental se constituye como un elemento fundamental de la salud en general; es un estado de completo bienestar físico, mental y social; no es solamente la ausencia de afecciones o enfermedades. Con una buena salud mental la persona es capaz de hacer frente al estrés cotidiano, de trabajar de forma productiva y de contribuir a su comunidad (Organización Mundial de la Salud [OMS], 2004). Se ha reconocido al ser humano como un ser social, que necesita relacionarse con los que lo rodean y sentirse bien en dicho contexto; por lo mencionado, cualquier factor natural o de carácter voluntario e involuntario que se presente en el medio pueden influenciar de manera positiva o negativa en la salud del individuo. Entonces, las emociones se establecen como una parte esencial de la vida cotidiana del sujeto y es un elemento clave para relacionarse con su entorno.

Revisando literatura se ha identificado que existe mayor énfasis en el estudio de emociones negativas que en el estudio de las emociones las positivas. Las emociones negativas, por ejemplo, el enojo, genera diversas reacciones que posiblemente llevarán a acciones, estos procesos dependen del propósito de la persona y dependerá exclusivamente de ella llevarlo a cabo. El autocontrol que tenga el sujeto, además de la forma en que resuelve sus problemas en el contexto social forman parte de sus estilos de defensa. Los modelos de esas emociones hacen énfasis en el rol de acciones a realizarse y las funciones adaptativas de las emociones se adaptan a las situaciones que representen riesgos (Greco, 2010). En tal sentido Werneck y Navarro (2011) indican que los resultados sobre el estudio de niveles de actividad física y estado de ánimo en adolescentes reflejaron aspectos positivos. Es decir, el ejercicio es un factor importante tanto en el desarrollo físico como psicológico de los adolescentes, es decir, una motivación auto determinada tanto en la actividad física como en el deporte hacia la construcción de valores cognitivos y afectivos (Bonilla et al.,2020), los cuales debería ser estimulado desde edades tempranas, tomando en cuenta que aproximadamente a los 16 años los niveles de ejercicio físico disminuyen gradualmente. En diferentes estudios se 
han verificado los beneficios que conlleva el ejercicio físico en la ansiedad y la depresión en jóvenes, demostrando un efecto positivo de la combinación del ejercicio junto con el tratamiento psicológico y farmacológico. Es importante resaltar que la ansiedad como estado puede estar ligada a una situación determinada, mientras que la ansiedad como rasgo está ligada a aspectos personales de cada persona que se encuentra definida por su personalidad. Como argumenta Vaca y Mayorga (2021), los episodios de ansiedad afectan la calidad del sueño debido a que los niveles elevados de estrés incurren en una mayor activación psicológica y fisiológica lo desencadena en sueño corto y fragmentado. Como lo hace notar Ramón et al. (2017), el ejercicio físico practicado de manera moderada si disminuye los efectos de la ansiedad brindando a la persona una sensación de bienestar, mejorando su estado de ánimo y la autoestima, se añade también que ayuda a la calidad del sueño.

Se ha demostrado que el ejercicio físico ayuda a las personas con trastornos del sueño siendo así una alternativa ante el consumo de medicamentos con el mismo fin. También argumentan que el ejercicio físico mejora el estado de ánimo, permite la regulación de la función cognitiva y previene el desarrollo de demencia, esto debido al aumento en el flujo sanguíneo hacia áreas del sistema nervioso central involucradas con el aprendizaje y otras funciones mentales. En la investigación de Cooney et al. (2014), a través de pruebas controladas aleatorias, relacionaron la práctica de ejercicio físico en tratamiento estándar y la ausencia de tratamiento utilizando un tratamiento placebo. La población de estudio estaba conformada por personas mayorea de 18 años. Al comparar los datos de las personas bajo el tratamiento con los de la población que no lo recibió, los resultados arrojaron que el ejercicio físico si tiene influencia en la prevención del desarrollo de cuadros de depresión. Entre las características clínicas de la depresión se encuentran problemas de alimentación, declive de la salud, tristeza, ideas o intentos autolíticos e insomnio (Sailema \& Mayorga, 2021).

Por otra parte McMahon et al. (2017) analizaron la relación que existe entre los niveles de actividad física y procesos ansiosos. La población de estudio fueron 11000 jóvenes de origen europeo de 10 países, hombres y mujeres. Los resultados que arrojó la investigación fueron que mientras más ejercicio físico que realice una persona, la probabilidad de padecer problemas psicológicos como ansiedad disminuye. Los investigadores de ese estudio argumental que los gobiernos debería ser implementar como políticas de estado actividades relacionadas al deporte y para fortalecer el desarrollo de adolescentes y jóvenes activos para disminuir el porcentaje de personas sedentarias con posibles complicaciones en la salud. Por su parte Wang et al. (2014) en su estudio sobre la influencia del ejercicio físico en el abusos de sustancias, concluyen que la práctica de ejercicio físico además de reducir los niveles de ansiedad y depresión, podrían provocar abstinencia en el uso de dichas sustancias. Con relación al tipo de sustancias, los puntos más notables fueron en el consumo de alcohol y drogas ilegales, y los puntos más bajos 
al exceso de uso de tabaco. Otros estudios en los que se incluían diferentes tipos de ejercicios, así como intensidad y períodos de ejecución de los individuos, se asemejan en los resultados, donde se manifiesta que el ejercicio aeróbico en distintas intensidades contribuye en el tratamiento de desórdenes mentales a los que son propensos los jóvenes. En este contexto, el presente documento muestra el resultado de un estudio realizado para determinar la relación entre ejercicio físico y el estado de ánimo de los estudiantes de bachillerato de la Unidad Educativa 17 de abril de la ciudad de Ambato durante el periodo abril-agosto 2021.

\section{Metodología}

Se trata de una investigación con enfoque cuantitativo porque se realizó un análisis estadístico a los datos recopilados. Los instrumentos de recolección de datos se plantearon de acuerdo con los objetivos establecidos. Los resultados permitieron la verificación de una de las hipótesis previamente planteadas. Al no experimentar con la población, es decir no hubo intervención por parte del investigador, la investigación es no experimental. Se analizaron datos de las variables de estudio en un tiempo determinado sobre la población por lo que es de tipo transversal. Con alcance descriptivo al detallar las características de las variables de estudio. El método del nivel teórico es hipotético-deductivo.

La muestra de estudio estuvo conformada por 49 estudiantes, hombres y mujeres de entre 16 y 18 años, pertenecientes al segundo de bachillerato general unificado de la Unidad Educativa 17 de abril.

El instrumento utilizado para identificar el nivel de ejercicio físico de la población fue el cuestionario internacional de la actividad física (IPAQ) que permite identificar las actividades que el individuo realizó los últimos 7 días, esto a través de 7 preguntas. Para la valoración del nivel de ejercicio físico de cada participante en el estudio se utilizaron mets de acuerdo con los siguientes indicadores: menos de 600 mets= Bajo o nulo nivel de ejercicio físico. De 600 a 1499 mets= Moderado nivel de ejercicio físico. Finalmente, más de 1500 mets= Alto nivel de ejercicio físico. Los Mets se calculan de la siguiente manera: 8 mets * días de actividad física rigurosa * minutos al día de actividad física rigurosa +4 mets $*$ días de actividad física moderada $*$ minutos al día de actividad física moderada $+3,3$ mets $*$ días de acción de caminata $*$ minutos al día de acciones de caminata $=$ total mets).

En cuanto al estado de ánimo de los estudiantes, la herramienta utilizada fue el test de EVEA que consta de 16 preguntas en las que se analiza diferentes emociones y estados de ánimo. Para valoración de los ítems se emplea la escala de Likert de 0 a 10 en la que el sujeto seleccionará un valor de la escala, esto de acuerdo con cómo se siente en el momento en el que responde. Se suman los valores de los ítems 4, 7, 10 y 16 para el estado de ánimo triste-depresivo. Los ítems 1, 5, 9, 13 para el estado de ánimo ansioso. 
Los ítems 3, 6, 12 y 15 para el estado de ánimo ira-hostilidad, y los ítems 2, 8, 11 y 14 para el estado de ánimo alegría. Al finalizar se suman los ítems y el resultado se dividen para cuatro. Predomina el estado de estado de ánimo que tenga el valor más alto.

El tratamiento estadístico de los datos y resultados de la investigación se elaboraron a través del software estadístico SPSS versión 25 para Windows. De las variables cualitativas se analizaron frecuencias, porcentajes y se efectuó una interpretación de tipo descriptiva de la muestra de estudio.

Para la verificación de la hipótesis de investigación y diferenciación estadística general, se aplicó en primera instancia la prueba de normalidad de Shapiro-Wilk que determinó la aplicación de pruebas no paramétricas para muestras independientes ( $U$ de Mann Whitney) y la prueba Chi-cuadrado de Pearson para determinar la asociación entre las variables de estudio.

\section{Resultados}

Los resultados que se obtuvieron de los test fueron analizados a través del software estadístico SPSS de forma cuantitativa. Los análisis de los resultados representados en tablas se desarrollaron de acuerdo con los objetivos previamente establecidos reflejando el nivel de ejercicio físico de los estudiantes y su estado de ánimo. Se elaboró una tabla cruzada donde se pudo determinar la relación entre la variable dependiente e independiente.

En la tabla 1 se encuentran los datos numéricos de la muestra de estudio con la cantidad total de hombres, mujeres y el rango de edad de estos.

Tabla 1

Caracterización de la muestra de estudio

\begin{tabular}{lllllll}
\hline Sexo & $\mathrm{f}$ & $\%$ & Edad (años) & & \\
\cline { 5 - 7 } & & & Total & Mínimo & Máximo & DS \\
\hline Masculino & 23 & 46.9 & 23 & 16 & 18 & \pm 16.39 \\
Femenino & 26 & 53.1 & 26 & 16 & 18 & \pm 16.42 \\
\hline P & & & $0.812^{* *}$ & & & \\
\hline Total & 49 & 100.0 & 49 & 16 & 18 & \pm 16.41 \\
\hline
\end{tabular}

Una vez realizado el análisis estadístico de frecuencias (f) entre las variables de estudio sexo y edad, se determinó que no existe una diferencia significativa porque $\mathrm{P}$ valor $\geq 0.05$. 
Resultados de la valoración de la práctica de ejercicio físico de los estudiantes de bachillerato de la Unidad Educativa 17 de abril de la ciudad de Ambato durante el período abril - agosto 2021.

Aplicando la metodología propuesta para la investigación, a través del cuestionario internacional de actividad física IPAQ, se valoró los días y minutos de práctica de actividades físicas de carácter riguroso, moderado y acciones de caminata realizadas por los estudiantes en los últimos 7 días, lo cual permitió calcular los met's de manera individual y promedio grupal de la muestra de estudio, resultados que se evidencian en la tabla 2.

Tabla 2

Cálculo de met's por práctica de ejercicio física rigurosa, moderadas y acciones de caminata

\begin{tabular}{|c|c|c|c|c|c|c|c|c|}
\hline $\begin{array}{l}\text { Variables } \\
\text { IPAQ }\end{array}$ & & Sexo & $\mathrm{N}$ & Mínimo & Máximo & Media & $\begin{array}{l}\text { Desv. } \\
\text { Desviación }\end{array}$ & $P$ \\
\hline \multirow{3}{*}{$\begin{array}{l}\text { Días } \\
\text { actividades } \\
\text { físicas } \\
\text { rigurosas }\end{array}$} & \multirow[t]{3}{*}{ de } & Masculino & 23 & 0 & 5 & 2.83 & 1.800 & \multirow[t]{3}{*}{$0.004^{*}$} \\
\hline & & Femenino & 26 & 0 & 4 & 1.38 & 1.359 & \\
\hline & & Total & 49 & 0 & 5 & 2.06 & 1.725 & \\
\hline \multirow{3}{*}{$\begin{array}{l}\text { Minutos } \\
\text { actividades } \\
\text { físicas } \\
\text { rigurosas }\end{array}$} & \multirow[t]{3}{*}{ de } & Masculino & 23 & 0 & 180 & 51.52 & 44.041 & \multirow[t]{3}{*}{$0.003^{*}$} \\
\hline & & Femenino & 26 & 0 & 90 & 19.62 & 24.038 & \\
\hline & & Total & 49 & 0 & 180 & 34.59 & 38.063 & \\
\hline \multirow{3}{*}{$\begin{array}{l}\text { Días } \\
\text { actividades } \\
\text { físicas } \\
\text { moderadas }\end{array}$} & \multirow[t]{3}{*}{ de } & Masculino & 23 & 0 & 7 & 2.65 & 1.555 & \multirow[t]{3}{*}{$0.307^{* *}$} \\
\hline & & Femenino & 26 & 0 & 5 & 2.15 & 1.690 & \\
\hline & & Total & 49 & 0 & 7 & 2.39 & 1.631 & \\
\hline \multirow{3}{*}{$\begin{array}{l}\text { Minutos } \\
\text { actividades } \\
\text { físicas } \\
\text { moderadas }\end{array}$} & \multirow[t]{3}{*}{ de } & Masculino & 23 & 0 & 90 & 41.74 & 22.136 & \multirow[t]{3}{*}{$0.010^{*}$} \\
\hline & & Femenino & 26 & 0 & 60 & 26.54 & 17.820 & \\
\hline & & Total & 49 & 0 & 90 & 33.67 & 21.183 & \\
\hline \multirow{3}{*}{$\begin{array}{l}\text { Días } \\
\text { acciones } \\
\text { caminata }\end{array}$} & \multirow{3}{*}{$\begin{array}{l}\text { de } \\
\text { de }\end{array}$} & Masculino & 23 & 0 & 7 & 3.91 & 1.952 & \multirow[t]{3}{*}{$0.887 * *$} \\
\hline & & Femenino & 26 & 0 & 7 & 4.04 & 2.375 & \\
\hline & & Total & 49 & 0 & 7 & 3.98 & 2.165 & \\
\hline \multirow{3}{*}{$\begin{array}{l}\text { Minutos } \\
\text { acciones } \\
\text { caminata }\end{array}$} & \multirow{3}{*}{$\begin{array}{l}\text { de } \\
\text { de }\end{array}$} & Masculino & 23 & 0 & 120 & 36.74 & 23.674 & \multirow[t]{3}{*}{$0.169 * *$} \\
\hline & & Femenino & 26 & 0 & 120 & 31.92 & 29.802 & \\
\hline & & Total & 49 & 0 & 120 & 34.18 & 26.933 & \\
\hline
\end{tabular}




\section{Tabla 2}

Cálculo de met's por práctica de ejercicio física rigurosa, moderadas y acciones de caminata (continuación)

\begin{tabular}{llllllll}
\hline $\begin{array}{l}\text { Variables } \\
\text { IPAQ }\end{array}$ & Sexo & N & Mínimo & Máximo & Media & $\begin{array}{l}\text { Desv. } \\
\text { Desviación }\end{array}$ & P \\
\hline METS & Masculino & 23 & 159 & 5720 & 2371.83 & 1572.633 & $0.006^{*}$ \\
& Femenino & 26 & 0 & 3172 & 1211.15 & 897.214 & \\
\cline { 2 - 5 } & Total & 49 & 0 & 5720 & 1755.96 & 1376.707 & \\
\hline
\end{tabular}

$\mathrm{P} \leq 0.05(*), \mathrm{P} \geq 0.05(* *)$

En base al cálculo de los met's, se categorizó a la muestra de estudio en los diferentes niveles planteados por el mismo instrumento y especificados en la metodología de la investigación (tabla 3)

Tabla 3

Niveles de práctica de ejercicios físicos en base al IPAQ

\begin{tabular}{lllllll}
\hline & \multicolumn{2}{l}{ Masculino } & \multicolumn{2}{c}{ Femenino } & \multicolumn{2}{c}{ Total } \\
\cline { 2 - 7 } Niveles de ejercicios físico & $\mathrm{f}$ & $\%$ & $\mathrm{f}$ & $\%$ & $\mathrm{f}$ & $\%$ \\
\hline Nivel alto & 16 & 69.6 & 8 & 30.8 & 24 & 49.0 \\
Nivel moderado & 3 & 13.0 & 11 & 42.3 & 14 & 28.6 \\
Nivel bajo o inactivo & 4 & 17.4 & 7 & 26.9 & 11 & 22.4 \\
\hline Total & 23 & 100.0 & 26 & 100.0 & 49 & 100.0 \\
\hline
\end{tabular}

Del $100 \%$ de sujetos pertenecientes al género masculino el $69.6 \%$ tiene un nivel alto de ejercicio físico, el $13 \%$ presenta nivel moderado de ejercicio físico y el $17.4 \%$ nivel bajo o inactivo.

En cuanto a los sujetos del género femenino, del $100 \%$ el $30.8 \%$ presenta un nivel alto de ejercicio físico, el $42.3 \%$ tiene nivel moderado de ejercicio físico y el $26.9 \%$ nivel bajo de ejercicio o inactivo.

Es decir, en el género masculino el nivel de ejercicio físico es alto y en el género femenino el nivel de ejercicio es moderado y bajo o inactivo.

Resultados de la identificación del estado de ánimo de los estudiantes de bachillerato de la Unidad Educativa 17 de abril de la ciudad de Ambato durante el periodo abril-agosto 2021. 
Mediante el uso del instrumento EVEA se determinaron los estados de ánimo de los sujetos. Para ello se valoraron diferentes coeficientes con base en los estados propuestos cuyos resultados se evidencia en la tabla 4.

\section{Tabla 4}

Coeficientes con base en los estados de ánimo en base a EVEA

\begin{tabular}{|c|c|c|c|c|c|c|c|c|c|c|}
\hline \multirow{2}{*}{$\begin{array}{l}\text { Coeficientes de } \\
\text { estados de ánimo }\end{array}$} & \multicolumn{3}{|c|}{ Masculino } & \multicolumn{3}{|c|}{ Femenino } & \multirow[t]{2}{*}{$\mathrm{P}$} & \multicolumn{3}{|c|}{ Total } \\
\hline & $\mathrm{n}$ & M & DS & $\mathrm{n}$ & M & DS & & $\mathrm{n}$ & M & DS \\
\hline Triste-Depresivo & 23 & 3.24 & 2.744 & 26 & 3.42 & 2.665 & $0.779 * *$ & 49 & 3.34 & 2.676 \\
\hline Ansiedad & 23 & 2.80 & 2.440 & 26 & 2.95 & 2.468 & $0.771 * *$ & 49 & 2.88 & 2.430 \\
\hline Ira-Hostilidad & 23 & 1.98 & 1.862 & 26 & 2.46 & 2.343 & $0.644 * *$ & 49 & 2.23 & 2.123 \\
\hline Alegre & 23 & 5.83 & 2.238 & 26 & 5.20 & 2.728 & $0.127 * *$ & 49 & 5.49 & 2.504 \\
\hline
\end{tabular}

El cálculo de los coeficientes permitió identificar la predominancia de uno u otro y de esta manera categorizar a la muestra de estudio en los siguientes estados de ánimo (tabla $5)$.

\section{Tabla 5}

Categorización de los estados de ánimo de la muestra de estudio

\begin{tabular}{lllllll}
\hline & \multicolumn{2}{l}{ Masculino } & \multicolumn{2}{l}{ Femenino } & \multicolumn{2}{l}{ Total } \\
\cline { 2 - 7 } Estados de Ánimo & $\mathrm{f}$ & $\%$ & $\mathrm{f}$ & $\%$ & $\mathrm{f}$ & $\%$ \\
\hline Triste-Depresivo & 4 & 17.4 & 6 & 23.1 & 10 & 20.4 \\
Ansiedad & 1 & 4.3 & 1 & 3.8 & 2 & 4.1 \\
Ira-Hostilidad & 0 & 0 & 1 & 3.8 & 1 & 2.0 \\
Alegría & 18 & 78.3 & 18 & 69.2 & 36 & 73.5 \\
\hline Total & 23 & 100.0 & 26 & 100.0 & 49 & 100.0 \\
\hline
\end{tabular}

Se puede identificar un mayor porcentaje en la escala de triste-depresivo en el género femenino con el $23.1 \%$, mientras que en la escala ansiedad tenemos el mismo número de sujetos en el género masculino y femenino, en cuanto a la escala ira-hostilidad se identificó a 1 solo sujeto correspondiente al 3.8\% de estudio del género femenino. En la escala alegría se identifica un mayor porcentaje, el 78.3\%, en el género masculino. En general identificamos que la escala de alegría es en la que se encuentra la mayor cantidad de la población, estando muy por encima de los estados de ánimo negativos que son tristedepresivo, ansiedad e ira-hostilidad. 
Resultados del análisis de la relación entre la entre la práctica de ejercicio físico y el estado de ánimo de los estudiantes de bachillerato de la Unidad Educativa 17 de abril de la ciudad de Ambato durante el periodo abril - agosto 2021.

Una vez identificado el nivel de ejercicio físico a través del (IPAQ) y el estado de ánimo por medio del (EVEA) se realizó una tabla cruzada la cual ayuda a describir si hay una relación entre niveles de ejercicio alto y moderado con estados de ánimo positivos y niveles bajos o inactivos con estados de ánimo negativos.

\section{Tabla 6}

Tabla cruzada de niveles de ejercicio físico y estados de ánimo.

\begin{tabular}{|c|c|c|c|c|c|c|}
\hline \multirow[t]{2}{*}{ Género } & \multirow{2}{*}{$\begin{array}{l}\text { Niveles de } \\
\text { ejercicio físico }\end{array}$} & \multicolumn{4}{|c|}{ Estado de Animo } & \multirow[t]{2}{*}{ Total } \\
\hline & & $\begin{array}{c}\text { Triste- } \\
\text { Depresivo }\end{array}$ & Ansiedad & $\begin{array}{c}\text { Ira- } \\
\text { Hostilidad }\end{array}$ & Alegre & \\
\hline \multirow[t]{4}{*}{ Masculino } & Nivel Alto & 0 & 1 & 0 & 15 & 16 \\
\hline & Nivel Moderado & 0 & 0 & 0 & 3 & 3 \\
\hline & Nivel Bajo o Inactivo & 4 & 0 & 0 & 0 & 4 \\
\hline & Total & 4 & 1 & 0 & 18 & 23 \\
\hline \multirow[t]{4}{*}{ Femenino } & Nivel Alto & 0 & 0 & 0 & 8 & 8 \\
\hline & Nivel Moderado & 0 & 0 & 1 & 10 & 11 \\
\hline & Nivel Bajo o Inactivo & 6 & 1 & 0 & 0 & 7 \\
\hline & Total & 6 & 1 & 1 & 18 & 26 \\
\hline \multirow[t]{4}{*}{ Total } & Nivel Alto & 0 & 1 & 0 & 23 & 24 \\
\hline & Nivel Moderado & 0 & 0 & 1 & 13 & 14 \\
\hline & Nivel Bajo o Inactivo & 10 & 1 & 0 & 0 & 11 \\
\hline & Total & 10 & 2 & 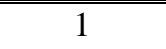 & 36 & 49 \\
\hline
\end{tabular}

De los 23 estudiantes de género masculino, 16 se encuentran con un nivel de ejercicio físico alto, 15 de ellos presentan un estado de ánimo de alegría, mientras 1 tiene un estado de ánimo de ansiedad. De 3 estudiantes se encuentran con un nivel de ejercicio físico moderado, todos presentan un estado de ánimo de alegría. Los 4 estudiantes restantes tienen un nivel de ejercicio físico bajo o inactivo y presentan el estado de ánimo tristedepresivo.

En cuanto a las 26 estudiantes de género femenino, 8 tienen un nivel de ejercicio físico alto y estado de ánimo de alegría. 11 estudiantes tienen un nivel de ejercicio físico moderado de las cuales 10 presentan un estado de ánimo de alegría mientras que el 1 restante tiene un estado de ánimo de ira-hostilidad. De las 7 estudiantes restantes, 6 de ellas presentan un estado de ánimo triste-depresivo y 1 un estado de ánimo de ansiedad.

Mediante la aplicación de las pruebas estadísticas seleccionadas para la comparar significación y asociación entre las variables, se pueden identifican los siguientes resultados (tabla 7). 


\section{Tabla 7}

Análisis estadístico de significación y asociación entre variables de estudio

\begin{tabular}{|c|c|c|c|c|}
\hline \multicolumn{5}{|c|}{ Pruebas de chi-cuadrado } \\
\hline Sexo & Pruebas estadísticas & Valor & $\mathrm{df}$ & $\begin{array}{l}\text { Significación asintótica } \\
\text { (bilateral) }\end{array}$ \\
\hline \multirow[t]{2}{*}{ Masculino } & Chi-cuadrado de Pearson & 23.240 & 4 & $0.000 *$ \\
\hline & $\mathrm{N}$ de casos válidos & 23 & & \\
\hline \multirow[t]{2}{*}{ Femenino } & Chi-cuadrado de Pearson & 27.051 & 6 & $0.000 *$ \\
\hline & $\mathrm{N}$ de casos válidos & 26 & & \\
\hline \multirow[t]{2}{*}{ Total } & Chi-cuadrado de Pearson & 48.725 & 6 & $0.000 *$ \\
\hline & N de casos válidos & $\overline{49}$ & & \\
\hline
\end{tabular}

Para aplicar el análisis de relación entre las variables de estudio se utilizó la prueba estadística de Chi-cuadrado de Pearson mediante la cual se determinó que en el grupo de género masculino el nivel de confiabilidad del 99\% (0.01) con 4 grados de libertad. Dando como resultado un valor límite de aceptación de 13.277. Por su parte en el grupo de género femenino presenta el mismo nivel de confiabilidad con 6 grados de libertad. El valor límite de aceptación es de 16.812. En los resultados del análisis se encontró en el género masculino un valor de 23.240 y en el género femenino un valor de 27.051. En el total de la muestra de estudio el valor es de 48.725. Los valores se encuentran en la zona de aceptación. Los resultados de la investigación fueron comprobados con diferenciación asintótica bilateral en un nivel de $\mathrm{P} \leq 0.05$ dando como resultado un valor estadístico significativo en el nivel de asociación entre las dos variables de investigación comprobándose la hipótesis alternativa, es decir que la práctica del ejercicio físico está asociada con el estado de ánimo de los estudiantes.

\section{Discusión}

Los resultados del presente estudio verificaron que los sujetos que presentan un nivel de ejercicio físico entre moderado y alto, experimentan estados de ánimos positivos, mientras que los sujetos que tienen un nivel de actividad física bajo o inactivo experimentan estados de ánimo negativos tales como depresión o ansiedad.

El bienestar psicológico está vinculado con la práctica de actividad física. Los individuos que realizan ejercicio regularmente se sienten más saludables, menos estresados y con mejor estado de ánimo que aquellas personas que no realizan ninguna actividad, presentando tristeza, fatiga y menor vigor. Otro resultado importante de esta investigación y que se asemeja con el presente estudio es que las mujeres perciben mayores estados de 
ánimo negativos. Así mismo Bonet et al. (2017) concluyeron que la práctica de ejercicio físico mejora el estado de ánimo de las personas activas como también de las no activas con regularidad, pero recalcando que los sujetos que si realizaban ejercicio físico con regularidad tenían un mejor estado de ánimo. En la investigación antes mencionada se considera necesario tener en consideración el género, el nivel de ejercicio diferenciando a las personas por activas y no activas, esto con la finalidad de recomendar el tipo, la duración, intensidad y la frecuencia del ejercicio físico a realizar ideal para cada sujeto. Por su parte Olmedilla et al. (2010) realizaron un estudio sobre la ansiedad, depresión y práctica de ejercicio físico en estudiantes universitarias. Los resultados demostraron que las estudiantes que practican actividad física sean o no federadas, tienen menores niveles de ansiedad y depresión que las estudiantes sedentarias, aunque en el estudio las diferencias con la ansiedad no han resultado significativas en lo estadístico, sí es notable que la práctica de ejercicio físico se relaciona con niveles más bajos de ansiedad. Finalmente, Vallejo y Zuleta ( 2019) también concuerdan con los resultados del presente trabajo investigativo donde se establece que realizar cualquier ejercicio físico disminuye las probabilidades de experimentar depresión y ansiedad; es decir, a mayor inactividad física del escolar a la semana, estos serán más propensos a padecer estados de ánimo negativos.

\section{Conclusiones}

- La información obtenida en el presente estudio demuestra que los estudiantes de la Unidad Educativa 17 de abril que presentan estados de ánimo positivos son aquellos que tienen un nivel alto y moderado de ejercicio físico, mientras que los jóvenes que son sedentarios, es decir, que tienen un nivel de ejercicio físico bajo de ejercicio físico experimentan estados de ánimo negativos.

- Se analizó el nivel de ejercicio físico que realizan los estudiantes de la Unidad Educativa 17 de abril tanto en hombres como en mujeres, cuyos datos reflejaron que el nivel de ejercicio físico de la mayoría oscila entre moderado y alto. No obstante, una parte de la población estudiada es considerada sedentaria porque su nivel de ejercicio físico es bajo o nulo.

- Al analizar las dos variables de estudio se establece que si existe relación entre el nivel de ejercicio físico y el estado de ánimo en los sujetos de estudio.

- Es importante destacar que existe una diferenciación entre géneros. Es así como los sujetos pertenecientes al género femenino tienen un nivel de ejercicio físico de moderado a bajo, por el contrario, los sujetos de género masculino, en su mayoría presentar un nivel alto de ejercicio físico. Además, los sujetos de género femenino presentan un mayor índice de emociones negativas (triste-depresivo) que el género masculino. 


\section{Referencias Bibliográficas}

Bisquert Bover, M., Ballester Arnal, R., Gil Llario, M. D., Elipe Miravet, M., \& López Fando Galdón, M. (2020). Motivaciones para el ejercicio físico y su relación con la salud mental y física: un análisis desde el género. Revista INFAD de Psicología. International Journal of Developmental and Educational Psychology., 1(1), 351-360. https://doi.org/10.17060/ijodaep.2020.n1.v1.1792

Bonet, J., Parrado, E., \& Capdevila, L. (2017). Acute effects of exercise on mood and HRV. Acute Effects of Exercise on Mood and HRV, 17(65), 85-100. https://doi.org/10.15366/rimcafd2017.65.006

Bonilla, J. A. M., Benítez, M. C., \& Galak, E. L. (2020). Apoyo motivacional docente y necesidades psicológicas básicas en la Educación Física: una revisión sistemática. Ciencia Digital, 4(1.1.), 5-20. https://doi.org/10.33262/cienciadigital.v4i1.1.1160

Cooney, G. M., Dwan, K., Greig, C. A., Lawlor, D. A., Rimer, J., Waugh, F. R., McMurdo, M., \& Mead, G. E. (2014). Exercise for depression. Advances in Psychiatric Treatment, 20(1), 2. https://doi.org/10.1192/apt.20.1.2

Greco, C. (2010). Las Emociones Positivas: Su Importancia En El Marco De La Promoción De La Salud Mental En La Infancia/ the Importation of Positive Emotions on Children'S Mental Health Promotion. Liberabit, 16(1), 81-93.

McMahon, E. M., Corcoran, P., O’Regan, G., Keeley, H., Cannon, M., Carli, V., Wasserman, C., Hadlaczky, G., Sarchiapone, M., Apter, A., Balazs, J., Balint, M., Bobes, J., Brunner, R., Cozman, D., Haring, C., Iosue, M., Kaess, M., Kahn, J.-P. Wasserman, D. (2017). Physical activity in European adolescents and associations with anxiety, depression and well-being. European Child \& Adolescent Psychiatry, 26(1), 111-122. https://doi.org/10.1007/s00787-016-0875-9

Olmedilla, A., Ortega, E., \& Candel, N. (2010). Ansiedad, depresión y práctica de ejercicio físico en estudiantes universitarias. Apunts Medicina de l'Esport, 45(167), 175-180. https://doi.org/10.1016/j.apunts.2010.03.001

Organización Mundial de la Salud [OMS]. (2004). Invertir en salud mental. Disponible en línea en: https://apps.who.int/iris/handle/10665/42897

Ramón, J., Santiesteban, G., Manuel, I., Cruz, G., Melvin, I., Plaza, Z., Singre Álvarez, J., Campoverde, D. G., Romero, E., \& Ii, F. (2017). Relación entre ansiedad y ejercicio físico. Revista Cubana de Investigaciones Biomédicas, 36(2), 169-177. http://scielo.sld.cu

Sailema, V., \& Mayorga, D. (2021). “La Depresión en adultos mayores La Depresión en 
adultos mayores por Covid-19 durante la emergencia sanitaria: Depression in older adults by Covid-19 during the health emergency. La U Investiga, 8(1), 107-116.

Tobar, K. D. L., Bonilla, J. A. M., \& Acosta, W. C. (2020). Análisis de la frecuencia cardíaca: un estudio con estudiantes universitarios que practican actividad física regular. Ciencia Digital, 21-31. https://doi.org/10.33262/cienciadigital.v4i1.1.1161

Vaca, D., \& Mayorga, D. (2021). Estados emocionales y su relación con el insomnio en el personal de salud durante pandemia. Enfermería Investiga, 6(1), 20-26.

Vallejo, A. P., \& Zuleta, K. M. P. (2019). Depresión, Ansiedad y Actividad Física en Escolares: Estudio Comparado. [Depression, anxiety and physical activity in school children: Comparative study.]. Revista Iberoamericana de Diagnóstico y Evaluación Psicologica, 52(3), 143-155.

Wang, D., Wang, Y., Wang, Y., Li, R., \& Zhou, C. (2014). Impact of physical exercise on substance use disorders: A meta-analysis. PLoS ONE, 9(10). https://doi.org/10.1371/journal.pone.0110728

Werneck, F. Z., \& Navarro, C. A. (2011). Nível de atividade física e estado de humor em adolescentes. Psicologia: Teoria e Pesquisa, 27(2), 189-193. https://doi.org/10.1590/s0102-37722011000200010

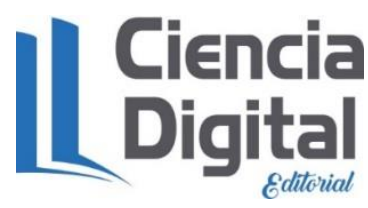


El artículo que se publica es de exclusiva responsabilidad de los autores y no necesariamente reflejan el pensamiento de la Revista Conciencia Digital.

\section{Ciencia \\ LDigital}

El artículo queda en propiedad de la revista y, por tanto, su publicación parcial y/o total en otro medio tiene que ser autorizado por el director de la Revista Conciencia Digital.

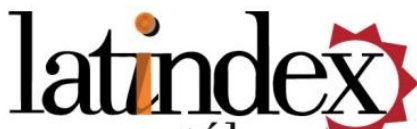

catálogo

ठ

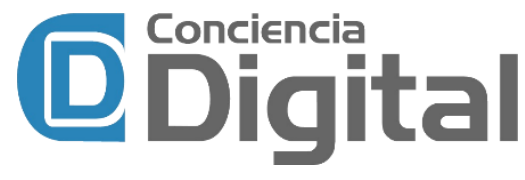

Indexaciones

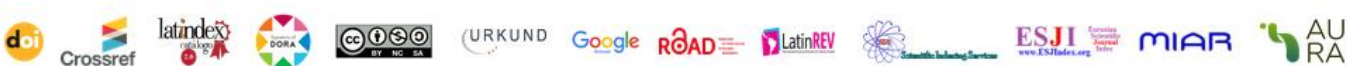

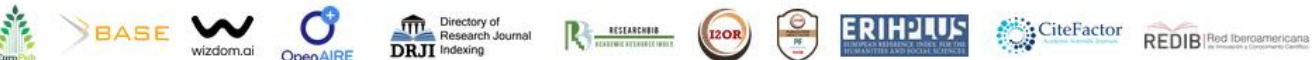

